International Mathematical Forum, 1, 2006, no. 30, 1453 - 1458

\title{
Inner invariant means on a locally compact group
}

\author{
Ali Ghaffari \\ Department of Mathematics, Semnan University, \\ Semnan University, Semnan, Iran
}

\begin{abstract}
For a locally compact group $G, L^{1}(G)$ is its group algebra and $L^{\infty}(G)$ is the dual of $L^{1}(G)$. In this paper, among the other things, we obtain a necessary and sufficient condition for $L^{\infty}(G)$ to have an inner invariant mean.
\end{abstract}

\section{Mathematics Subject Classification: 43A60}

Keywords: Banach algebras, locally compact group, inner invariant mean, unimodular group

\section{Introduction}

Let $G$ denote a locally compact group with identity, left Haar measure $\lambda$ and modular function $\Delta$. Let $L^{p}(G), 1 \leq p \leq \infty$ have the usual meaning and

$$
P^{p}(G)=\left\{f \in L^{p}(G) ; f \geq 0,\|f\|_{p}=1\right\} .
$$

For every $\phi \in L^{1}(G)$, and $f \in L^{p}(G)$ the $\star-$ convolution $\phi \star f$,

$$
\phi \star f(x)=\int_{G} \Delta(y)^{\frac{1}{p}} f\left(y^{-1} x y\right) \phi(y) d y \quad(x \in G)
$$

exists and represents an element of $L^{p}(G)$ of norm $\|\phi \star f\|_{p} \leq\|\phi\|_{1}\|f\|_{p}$ (see $[10])$.

A mean $m$ on $L^{\infty}(G)$ is a positive linear functional on $L^{\infty}(G)$ satisfying $m(1)=1$. For a function $f: G \rightarrow C$, we put

$$
{ }_{x} f_{x}(y)=f\left(x^{-1} y x\right)
$$


for any $x, y \in G$. Following Effros [1] and Yuan [10], we say that $G$ is inner amenable if there exists a mean $m$ on $L^{\infty}(G)$ such that $m\left({ }_{x} f_{x}\right)=m(f)$ for all $x \in G$ and $f \in L^{\infty}(G)$.

Inner amenability is a considerably weaker condition on $G$ than amenability in the usual sense. The free group on two generators is an easily accessible example of a group which is not inner amenable. Amenable locally compact groups and [IN]-groups are inner amenable [4]. We shall follow Hewitt [3] and Yuan [10] for definitions and terminologies not explained here. The literature on inner amenability has grown substantially in recent years (see [5], [6], [7] and $[12])$.

\section{Main Results}

Now we state the following characterization Theorem of inner amenable groups.

Theorem 2.1. For a locally compact group $G$, the following conditions are equivalent:

(1) $G$ is an inner amenable group.

(2) There exists a net $\left(f_{\alpha}\right)$ in $P^{2}(G) \cap C_{C}(G)$ such that for every compact subset $K$ of $G$,

$$
\lim _{\alpha}\left|1-f_{\alpha} \star f_{\alpha}(x)\right|=0
$$

uniformly on $K$.

(3) For every compact subset $K$ of $G$ and $\delta, \epsilon>0$, there exist compact subsets $U$ and $N$ in $G$ such that $|U|>0,|N|<\delta$, and

$$
\frac{\left|x U x^{-1} \Delta U\right|}{|U|}<\epsilon
$$

for all $x \in K \backslash N$.

Proof. Assuming that $G$ is inner amenable. Let a compact subset $K$ of $G$ and $\epsilon \in(0,1)$ be given. By Theorem 1 in [10], there exists $h \in P^{2}(G)$ such that $\left\|\Delta(y)^{\frac{1}{2}}{ }_{y} h_{y}-h\right\|_{2}<\frac{\epsilon}{6}$ for all $y \in K$. By density of $C_{C}(G)$, we may determine $\phi \in C_{C}(G)$ such that $\|\phi-h\|_{2}<\frac{\epsilon}{6}$. For every $y \in K$,

$$
\begin{aligned}
\left\|\Delta(y)^{\frac{1}{2}}{ }_{y} \phi_{y}-\phi\right\|_{2} & \leq\left\|\Delta(y)^{\frac{1}{2}}{ }_{y} \phi_{y}-\Delta(y)^{\frac{1}{2}}{ }_{y} h_{y}\right\|_{2}+\left\|\Delta(y)^{\frac{1}{2}}{ }_{y} h_{y}-h\right\|_{2}+\|\phi-h\|_{2} \\
& =\|\phi-h\|_{2}+\left\|\Delta(y)^{\frac{1}{2}}{ }_{y} h_{y}-h\right\|_{2}+\|\phi-h\|_{2}<\frac{\epsilon}{2} .
\end{aligned}
$$


Let $f=\frac{\phi}{\|\phi\|_{2}}$. It is easy to see that

$$
\left\|\Delta(y)^{\frac{1}{2}} f_{y}-f\right\|_{2}<\epsilon
$$

whenever $y \in K$. For every $y \in K$, we have

$$
\begin{aligned}
\left|1-\int_{G} \Delta(y)^{\frac{1}{2}} f\left(y^{-1} x y\right) f(x) d x\right| & =\left|\int_{G} f(x) f(x)-\Delta(y)^{\frac{1}{2}} f\left(y^{-1} x y\right) f(x) d x\right| \\
& \leq \int_{G}|f(x)|\left|f(x)-\Delta(y)^{\frac{1}{2}} f\left(y^{-1} x y\right)\right| d x \\
& \leq\|f\|_{2}\left(\int_{G}\left|f(x)-\Delta(y)^{\frac{1}{2}} f\left(y^{-1} x y\right)\right|^{2} d x\right)^{\frac{1}{2}} \\
& =\left\|f-\Delta(y)^{\frac{1}{2}}{ }_{y} f_{y}\right\|_{2}<\epsilon .
\end{aligned}
$$

This shows that $|1-f \star f(y)|<\epsilon$ for all $y \in K$. Consequently for each pair $(K, \epsilon)$, where $K \subseteq G$ is compact and $\epsilon>0$, there is a $f_{(K, \epsilon)} \in P^{2}(G) \cap C_{C}(G)$ such that

$$
\left|1-f_{(K, \epsilon)} \star f_{(K, \epsilon)}(y)\right|<\epsilon
$$

for $y \in K$. Then we define the partial ordering on the index set as $(K, \epsilon) \leq$ $\left(K^{\prime}, \epsilon^{\prime}\right)$ if $K \subseteq K^{\prime}$ and $\epsilon \geq \epsilon^{\prime}$. Clearly $f_{(K, \epsilon)} \star f_{(K, \epsilon)}$ converges to 1 in the uniform topology on compacta. So, (1) implies (2).

For the converse, if $\epsilon>0$ and compact set $K \subseteq G$ are specified, there is some $f \in P^{2}(G) \cap C_{C}(G)$ such that

$$
|1-f \star f(y)|<\frac{\epsilon^{2}}{2} .
$$

for all $y \in K$. On the other hand, for every $y \in K,\|f\|_{2}=\left\|\Delta(y)^{\frac{1}{2}}{ }_{y} f_{y}\right\|_{2}=1$. Hence,

$$
\begin{aligned}
\left\|f-\Delta(y)^{\frac{1}{2}}{ }_{y} f_{y}\right\|_{2}^{2} & =\int_{G}\left|f(x)-\Delta(y)^{\frac{1}{2}}{ }_{y} f_{y}(x)\right|^{2} d x \\
& =\|f\|_{2}^{2}+\left\|\Delta(y)^{\frac{1}{2}}{ }_{y} f_{y}\right\|_{2}^{2}-2 \int_{G} \Delta(y)^{\frac{1}{2}}{ }_{y} f_{y}(x) f(x) d x \\
& =2-2 \int_{G} \Delta(y)^{\frac{1}{2}}{ }_{y} f_{y}(x) f(x) d x \\
& =2-2 f \star f(y)<\epsilon^{2} .
\end{aligned}
$$

This shows that $\left\|f-\Delta(y)^{\frac{1}{2}}{ }_{y} f_{y}\right\|_{2}<\epsilon$ for all $y \in K$. By Theorem 1 in [10], $G$ is inner amenable.

(1) implies (3). Let a compact subset $K$ of $G$ and $\delta, \epsilon>0$ be given. By Theorem 2 in [11] there exists a compact subset $U$ of $G$ such that $0<|U|$ and

$$
\frac{\left|a U a^{-1} \Delta U\right|}{|U|}<\epsilon
$$


whenever $a \in K$. Thus (1) implies (3).

Finally it suffices to show that (3) implies (1). Let $K \subseteq G$ be compact with left Haar measure $|K|>0$ and let $W=K K$. We may assume the unite in $G$ is in $K$. Let $M=\max \left\{\Delta\left(x^{-1}\right) ; x \in W\right\}$ and $\epsilon>0$. Now apply (3) to $\delta=\frac{|K|}{2}, \frac{\epsilon}{2 M}$ and compact set $W$. There exist compact subsets $U$ and $N$ in $G$ such that $0<|U|,|N|<\delta$ and

$$
\frac{\left|a U a^{-1} \Delta U\right|}{|U|}<\frac{\epsilon}{2 M}
$$

whenever $a \in W \backslash N$. By Theorem 7.3 in [9],

$$
(W \backslash N) \bigcap a(W \backslash N) \neq \emptyset
$$

for all $a \in K$. For every $a \in K$, there exists $x, y \in W \backslash N$ such that $a=y x^{-1}$. Thus,

$$
\begin{aligned}
\frac{\left|a U a^{-1} \Delta U\right|}{|U|} & =\frac{\left|y x^{-1} U x y^{-1} \Delta U\right|}{|U|}=\frac{\left|x^{-1} U x y^{-1} \Delta y^{-1} U\right|}{|U|} \\
& =\Delta\left(y^{-1}\right) \frac{\left|x^{-1} U x \Delta y^{-1} U y\right|}{|U|} \\
& \leq M\left(\frac{\left|x^{-1} U x \Delta U\right|}{|U|}+\frac{\left|y^{-1} U y \Delta U\right|}{|U|}\right)<\epsilon .
\end{aligned}
$$

By Theorem 2 in [11], $G$ is inner amenable.

We denote by $\Omega$ the family of all nonvoid compact subsets in $G$. Let also

$$
\Omega_{0}=\{K \in \Omega ;|K|>0\} .
$$

Let $\mu \in M(G)$ and $f \in L^{p}(G)$, we consider

$$
L_{\mu}: f \mapsto \mu * f \quad\left(\mu * f(x)=\int_{G} f\left(y^{-1} x\right) d \mu(y)\right)
$$

We know that $G$ is amenable if and only if $\left\|L_{\mu}\right\|=\|\mu\|$ for any $\mu \in M(G)$ (for details see [8], [9]).

Let $\psi \in L^{1}(G)$. Define $T_{\psi}: L^{p}(G) \rightarrow L^{p}(G)$ given by $T_{\psi}(f)=\psi \star f$. In the following Theorem, we give sufficient conditions about $G$ such that $\left\|T_{\psi}\right\|=1$ for all $\psi \in P^{1}(G)$.

Theorem 2.2. Let $p$ a real number such that $1 \leq p<\infty$, and let $p^{\prime}=\frac{p}{p-1}$ $\left(1^{\prime}=\infty\right)$. Let $\psi \in P^{1}(G)$. Then $\left\|T_{\psi}\right\|=1$ if any one of the following conditions hold: 
(1) $G$ is an inner amenable group.

(2) $\sup \left\{\inf \left\{\sup \left\{\frac{|U|^{\frac{1}{p}}|V|^{\frac{1}{p^{\prime}}}}{\left|a U a^{-1} \cap V\right|} \Delta(a)^{\frac{-1}{p}} ; a \in K\right\} ; U, V \in \Omega_{0}\right\} ; K \in \Omega\right\}=1$.

Proof. Assume $G$ to be inner amenable. By Theorem 2.2 in [2], $\left\|T_{\psi}\right\|=1$ for each $\psi \in P^{1}(G)$.

Now assume that $(2)$ holds. Let $\phi \in P^{1}(G) \cap C_{C}(G)$ with $K=\operatorname{supp} \phi$. Given $\epsilon>0$, by assumption there exist $U, V \in \Omega_{0}$ such that

$$
\sup \left\{\frac{|U|^{\frac{1}{p}}|V|^{\frac{1}{p^{\prime}}}}{\left|a U a^{-1} \cap V\right|} \Delta(a)^{\frac{-1}{p}} ; a \in K\right\}<1+\epsilon .
$$

Let $f=\left(\frac{1}{|U|^{\frac{1}{p}}}\right) 1_{U} \in P^{p}(G)$ and $g=\left(\frac{1}{|V|^{\frac{1}{p^{\prime}}}}\right) 1_{V} \in P^{p^{\prime}}(G)$. We have

$$
\begin{aligned}
\inf \left\{\Delta(a)^{\frac{1}{p}}\left|a U a^{-1} \bigcap V\right| ; a \in K\right\} & \leq \int_{K} \Delta(x)^{\frac{1}{p}}\left|x U x^{-1} \bigcap V\right| \phi(x) d x \\
& =|U|^{\frac{1}{p}}|V|^{\frac{1}{p^{\prime}}} \int_{G} \Delta(x)^{\frac{1}{p}} \frac{\left|x U x^{-1} \cap V\right|}{|U|^{\frac{1}{p}}|V|^{\frac{1}{p^{\prime}}} \phi(x) d x} \\
& =|U|^{\frac{1}{p}}|V|^{\frac{1}{p^{\prime}}} \int_{G} \phi(x) \int_{G} \Delta(x)^{\frac{1}{p}} f\left(x^{-1} y x\right) g(y) d y d x \\
& =|U|^{\frac{1}{p}}|V|^{\frac{1}{p^{\prime}}} \int_{G} \phi \star f(y) g(y) d y \\
& =|U|^{\frac{1}{p}}|V|^{\frac{1}{p^{\prime}}}(\phi \star f, g) \\
& \leq|U|^{\frac{1}{p}}|V|^{\frac{1}{p^{\prime}}}\|\phi \star f\|_{p}\|g\|_{p^{\prime}} \\
& \leq|U|^{\frac{1}{p}}|V|^{\frac{1}{p^{\prime}}}\left\|T_{\phi}\right\| .
\end{aligned}
$$

Hence

$$
1 \leq \sup \left\{\frac{|U|^{\frac{1}{p}}|V|^{\frac{1}{p^{\prime}}}}{\left|a U a^{-1} \bigcap V\right|} \Delta(a)^{\frac{-1}{p}} ; a \in K\right\}\left\|T_{\phi}\right\|<(1+\epsilon)\left\|T_{\phi}\right\| .
$$

As $\epsilon>0$ may be chosen arbitrary, we obtain $\left\|T_{\phi}\right\|=1$. It is easy to see that $\left\|T_{\psi}\right\|=1$ for any $\psi \in P^{1}(G)$.

\section{References}

[1] E. G. Effros, Property $\Gamma$ and inner amenability, Proc. Amer. Math. Soc, 47 (1975), 483-486.

[2] A. Ghaffari, A characterization of locally compact inner amenable groups, To appear. 
[3] E. Hewitt and K. A. Ross, Abstract Harmonic analysis, Vol. I, Springer Verlage, Berlin, 1963; Vol. II, Springer Verlage, Berlin, 1970.

[4] A. T. Lau and A. L. T. Paterson, Inner amenable locally compact groups, Trans. Amer. Math. Soc, 325 (1991), 155-169.

[5] V. Losert and H. Rindler, Conjugate invariant means, Colloq. Math, 15 (1987), 221-225.

[6] R. Memarbashi and A. Riazi, Topological inner invariant means, Studia Sci. Math. Hungar, 40 (2003), 293-299.

[7] R. Nasr-Isfahani, Inner amenability of Lau algebras, Arch. Math. (Brno), 37 (2001), 45-55.

[8] A. L. T. Paterson, Amenability, Amer. Math. Soc. Math. Survey and Monogrraphs 29, Providence, Rhode Island, 1988.

[9] J. P. Pier, Amenable locally compact groups, John Wiley And Sons, New York, 1984.

[10] C. K. Yuan, Conjugate convolutions and inner invariant means, J. Math. Anal. Appl, 157 (1991), 166-178.

[11] C. K. Yuan, On the structures of locally compact groups admitting inner invariant means, in Lecture Notes in Math., Vol. 1494, pp. 208-216, Springer-Verlag, New York/Berlin, 1991.

[12] C. K. Yuan, The existence of inner invariant means on $L^{\infty}(G)$, J. Math. Anal. Appl, 130 (1988), 514-524.

\section{Received: November 26, 2005}

